\title{
Dynamics Modeling and Simulation of Planar Serial Rigid-flexible Manipulators
}

\author{
Zhang Piaoshi, Liu Jubao, Xia Yuanqiang, Gao Sheng \\ School of Mechanical Science and Engineering, Northeast Petroleum University, Heilongjiang \\ Daqing 163318, China
}

Keywords: Rigid-Flexible; Serial Manipulator; Dynamics Modeling; Symbol Derivation

\begin{abstract}
Dynamics modeling and its Matlab symbol derivation are proposed for serial rigid-flexible manipulators in terms of Lagrange approach, which has the properties of clear structure and standard format. Deformation of flexible link is described by Finite Element Method (FEM), and motion of any node can be obtained on the basis of homogeneous coordinate and matrix transformation. Different types of rigid-flexible manipulators can be implemented by the modeling and derivation method.
\end{abstract}

\section{Introduction}

With the applications of rigid-flexible manipulators become more and more widely used in the field of engineering, researches on the manipulator go more and more deeply. In order to study dynamic problems of this type manipulators better, it is very important to study the general and efficient modeling method. Aiming at this problem, one modeling method is proposed for serial rigid-flexible manipulators in terms of Finite Element Method (FEM), which is in combination with Matlab symbolic operation and realize the symbol derivation of system dynamics model. For most types of serial rigid-flexible manipulators, symbolic expression of numerical computation of system dynamics model can be obtained by this modeling method.

\section{Dynamics modeling of serial rigid-flexible manipulators}

In this paper flexibility and rigidity two ingredients are considered simultaneously in the process of modeling and some models are simplified under the premise that no influence exerted on the essential characteristics of the whole system. Firstly assuming the macro manipulator only generate bending deformation, no shear and torsion deformation occurs. Secondly assuming the manipulator only generates bending deformation in the horizontal plane. Therefore, there is no need to consider the influence of gravity in the analysis.

There are $\mathrm{m}$ links which are defined by the connecting conditions of rotary joint, consist of a rigid links and $b$ flexible links. For the flexible link $i$, discrete it into $n_{i}$ units, length of each unit is $l_{i}$, any unit of links is represented by $i j$, $i$ is the number of the link, $j$ is the $j$ th unit of the link. The rigid-flexible 2-links manipulator system model shown in Fig.1. Fig.1 reveals the definitions of the local coordinates and the joint variables of rigid-flexible manipulators and the definitions of local deformation of flexible manipulators. The deformation of flexible manipulators is described by Finite Element Method. According to above-mentioned definitions, the second flexible manipulator is divided into $\mathrm{n}_{2}$ units, each of which contains 2 nodal points.

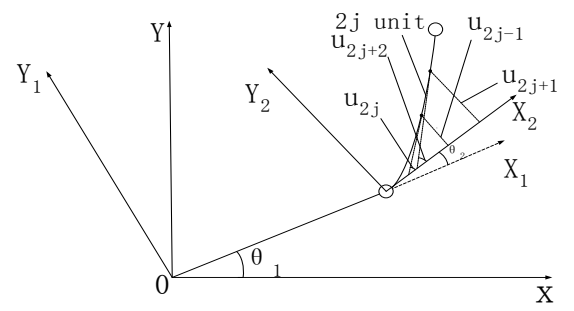

Fig.1. The model and parameters of serial rigid-flexible manipulators

Compared with the rigid manipulator, the motion of any point of the flexible manipulator can be 
divided into large range movement implicated in local coordinate system and superposition of flexible deformation which is relative to the local coordinate system. Therefore, its kinematic relations can be described by two coordinate conversion matrixes, respectively are:

$$
\begin{aligned}
T_{i 1} & =\left[\begin{array}{ccc}
\cos \theta_{i} & -\sin \theta_{i} & 0 \\
\sin \theta_{i} & \cos \theta_{i} & 0 \\
0 & 0 & 1
\end{array}\right] \\
T_{i 2} & =\left[\begin{array}{ccc}
\cos u_{2 n_{i}+2} & -\sin u_{2 n_{i}+2} & L_{i} \\
\sin u_{2 n_{i}+2} & \cos u_{2 n_{i}+2} & u_{2 n_{i}+1} \\
0 & 0 & 1
\end{array}\right]
\end{aligned}
$$

Among them, $\mathrm{T}_{\mathrm{i} 1}$ is used to describe the influence of joint angle on the attitude transformation, $\mathrm{T}_{\mathrm{i} 2}$ is used to describe the influence of flexible deformation on the attitude transformation. Value $\mathrm{u}_{2 \mathrm{ni}+1}$ and $\mathrm{u}_{2 \mathrm{ni}+2}$ of elastic deformation of $\mathrm{T}_{\mathrm{i} 2}$ can be set as 0 . In the formula, $\theta_{i}$ is the ith joint angle, $L_{i}$ is the length of the ith link $\left(L_{i}=n_{i} l_{i}\right), u_{2 n i+1}$ is the terminal transverse displacement of the ith link, $u_{2 n i+2}$ is the terminal elastic angle of the ith link.

In local coordinate system, any point $\mathrm{j}$ of the link i whose homogeneous coordinate is defined as:

$r_{i j}=\left[\begin{array}{lll}(j-1) l_{i}+x_{i j} & y_{i j} & 1\end{array}\right]^{T}$

Flexible deformation $\mathrm{y}_{\mathrm{ij}}$ can be described in the form of dirichlet series [1] as:

$$
y_{i j}\left(x_{i j}, t\right)=\sum_{k=1}^{4} \phi_{k}\left(x_{i j}\right) u_{2 j-2+k}(t)
$$

In the formula (4): $\phi_{k}$ is the dirichlet series equation, $\mathrm{u}_{2 \mathrm{j}-2+\mathrm{k}}$ is the generalized coordinates of the unit node. The world coordinates of the point can be computed as:

$$
{ }^{0} r_{i j}=T_{11} T_{12} T_{21} T_{22} \cdots T_{i 1} r_{i j}
$$

Speed of the point can be described as:

$$
\frac{\partial r}{\partial t}=\left[\begin{array}{ll}
\frac{\partial r}{\partial \theta} & \frac{\partial r}{\partial u}
\end{array}\right]\left[\begin{array}{c}
\dot{\theta} \\
\dot{u}
\end{array}\right]
$$

In the formula (6): $\theta$ is the variable array of the joint angle, $u$ is the elastic deformation array, both of them make up the generalized coordinate array of the system $q=[\theta \mathrm{u}]^{\mathrm{T}}$. The kinetic energy of any element ij of the link i can be described as:

$$
T_{i j}=\frac{1}{2} \int_{0}^{l_{i}} m_{i}\left[\frac{\partial r}{\partial t}\right]^{T}\left[\frac{\partial r}{\partial t}\right] d x_{i j}
$$

The potential energy can be described as:

$$
V_{i j}=\frac{1}{2} \int_{0}^{l_{i}} E I_{i}\left[\frac{\partial^{2} y_{i j}}{\partial x_{i j}{ }^{2}}\right]^{2} d x_{i j}
$$

For each unit $\mathrm{ij}$, work out the kinetic energy $T_{i j}$ and the potential energy $V_{i j}$ by use of generalized coordinates and generalized speed which describe the system motion, and then accumulate them to acquire the total kinetic energy and the total potential energy of the system [2] , that is

$$
\begin{aligned}
& T(q, \dot{q})=\sum_{i=1}^{m} \sum_{j=1}^{n_{i}} T_{i j} \\
& V(q)=\sum_{i=1}^{m} \sum_{j=1}^{n_{i}} V_{i j}
\end{aligned}
$$

In the formula (10): $\mathrm{m}$ is the amount of links. Thus system Lagrange function $L(q, \dot{q})=T(q, \dot{q})-V(q)$ can be obtained, then substituted them into Lagrange equation:

$\frac{d}{d t}\left[\frac{\partial L}{\partial \dot{\boldsymbol{q}}_{k}}\right]-\frac{\partial L}{\partial \boldsymbol{q}_{k}}=f_{k}, k=1,2, \ldots, n$

Dynamics model can be obtained after calculations as follows:

$$
M(q)\left[\begin{array}{l}
\ddot{\theta} \\
\ddot{u}
\end{array}\right]+H(q, \dot{q})\left[\begin{array}{l}
\dot{\theta} \\
\dot{u}
\end{array}\right]+K\left[\begin{array}{l}
\theta \\
u
\end{array}\right]=\left[\begin{array}{l}
\tau \\
0
\end{array}\right]
$$

In the formula (12): $\mathrm{M}$ is the system positive definite inertia matrix; $\mathrm{H}$ is one item that related to the Coriolis-exciting-force and the centripetal force; $K$ is the stiffness matrix; $\tau=[\tau 0]$ is input joint force array of manipulators. It must be pointed out that the damping force characteristic of the 
flexible manipulator is not evaluated in above formulas, because it is very complicated, the usual way to do this is to use the inertia matrix $\mathrm{M}$ and the stiffness matrix $\mathrm{K}$ to get it [3] .

\section{Matlab symbol derivation of dynamics model}

During symbolic operation, there is no need to assign values to variables, the operation results are expressed in standard symbolic form. In a certain sense, intuitive symbolic expression can be obtained no matter how complicated the operation relations are [4]. The following contents briefly explain some symbol commands. (1)syms: for creating symbolic variables and functions; (2)diff, int: for the integration and derivation of symbolic object; (3)subs: for the variable substitution and computation of the expression; The basic process is:

(1) According to the above figure, first defining system variables as symbolic variables by means of command syms. Such as syms $\begin{array}{cllllllll}T & V & T_{i j} & V_{i j} & r & J & x_{i j} & y_{i j}\end{array}$

(2) Make use of the matrix multiply and the command diff with formula (5) and formula (6) to acquire the position and the expression of velocity of any node on the link $\mathrm{i}$, then determine the kinetic energy $T_{i j}$ and the potential energy $V_{i j}$ of each element with formula (7) and formula (8). For examples, $\mathrm{T}_{\mathrm{ij}}=\operatorname{int}\left(1 / 2 * \mathrm{~m}_{\mathrm{i}} * \operatorname{diff}(\mathrm{r}, \mathrm{t}) .{ }^{*} \operatorname{diff}(\mathrm{r}, \mathrm{t}), \mathrm{x}_{\mathrm{ij}}, 0, \mathrm{~L}_{\mathrm{i}}\right)$ and $\mathrm{V}_{\mathrm{ij}}=\operatorname{int}\left(1 / 2 * \mathrm{E}_{\mathrm{i}} * \mathrm{I}_{\mathrm{i}} *\left(\operatorname{diff}\left(\mathrm{y}_{\mathrm{ij}}, \mathrm{x}_{\mathrm{ij}}, 2\right)\right) \wedge 2\right.$, $\left.\mathrm{x}_{\mathrm{ij}}, 0, \mathrm{~L}_{\mathrm{i}}\right)$;

(3) Acquire the total kinetic energy $\mathrm{T}$ and potential energy $\mathrm{V}$ of the system by means of accumulating the kinetic energy of unit $T_{i j}$ and potential energy of unit $V_{i j}$ and then obtain the Lagrangian of the system;

(4) Make use of command diff to substitute L into formula (11), proceed boundary condition procedure and then get item $\mathrm{M}, \mathrm{N}$ and $\mathrm{K}$ of formula (12) which is system dynamics model.

\section{Modeling and Simulation of 1-flex and 1-rigid manipulator}

Parameters of links are as follows: Length of link $L_{i}$ is $1 \mathrm{~m}$, mass $m_{i}$ is $2.473 \mathrm{~kg}$, section form of link is rectangle, inertia moment $I_{i}$ is $6 \times 10^{-9} \mathrm{~m}^{4}$, elasticity modulus $E=2.07 \times 10^{11} \mathrm{~Pa}$. For convenience, only discretize the flexible link to 1 unit and the wide range movement of system is known. The motion characteristics of each joint are the same which is shown in the formula (13).

$$
\theta_{i}=\left\{\begin{array}{cc}
\frac{\pi}{6}\left[\frac{2 t}{t_{0}}-\sin \left(\frac{4 \pi t}{t_{0}}\right) / 2 \pi\right], & 0 \leq t \leq \frac{t_{0}}{2} \\
\frac{\pi}{6}\left[2-\frac{2 t}{t_{0}}+\sin \left(\frac{4 \pi t}{t_{0}}\right) / 2 \pi\right], & t>\frac{t_{0}}{2}
\end{array}\right.
$$

The system generalized coordinates array is defined as $q=\left[\theta_{1}, \mathrm{u}_{1}, \mathrm{u}_{2}, \mathrm{u}_{3}, \mathrm{u}_{4}, \theta_{2}\right]^{\mathrm{T}}$. The boundary condition is $\mathrm{u}_{1}=\mathrm{u}_{2}=0$, that means flexible links are considered as the cantilever model [5]. Considered that characteristics of the wide range system movement are known, after boundary condition procedure, system generalized coordinates are translated into $q=\left[u_{3}, u_{4}\right]^{T} . u_{3}$ is transverse displacement of the endpoint of flexible links, $\mathrm{u}_{4}$ is elastic angle of the endpoint of flexible links. At this point, $\mathrm{M}_{1}(\mathrm{q})$ and $\mathrm{M}_{2}(\mathrm{q})$ are both $2 \mathrm{x} 2$ matrix, $\mathrm{H}$ is a $2 \mathrm{x} 1$ matrix. After Matlab symbol derivation, items of matrix $\mathrm{M}_{1}(\mathrm{q})$ are:

$$
\begin{array}{ll}
m_{11}=\frac{13}{35} m_{1} L_{1}+m_{2} L_{2} & m_{12}=-\frac{11}{210} m_{1} L_{1}^{2}+\frac{1}{2} m_{2} L_{2}^{2} \cos \left(u_{4}+\theta_{2}\right) \\
m_{21}=-\frac{11}{210} m_{1} L_{1}^{2}+\frac{1}{2} m_{2} L_{2}^{2} \cos \left(u_{4}+\theta_{2}\right) & m_{22}=\frac{1}{105} m_{1} L_{1}^{3}+\frac{1}{3} m_{2} L_{2}^{3}
\end{array}
$$

Items of matrix $\mathrm{M}_{2}(\mathrm{q})$ are:

$$
\begin{array}{ll}
m_{11}=\frac{7}{20} m_{1} L_{1}^{2}+\frac{1}{2} m_{2} L_{2}^{2} \cos \left(u_{4}+\theta_{2}\right)+m_{2} L_{1} L_{2} & m_{12}=\frac{1}{2} m_{2} L_{2}^{2} \cos \left(u_{4}+\theta_{2}\right) \\
m_{21}=-\frac{1}{20} m_{1} L_{1}^{3}+\frac{1}{2} m_{2} L_{2}^{2} u_{3} \sin \left(u_{4}+\theta_{2}\right)+\frac{1}{3} m_{2} L_{2}^{2}+\frac{1}{2} m_{2} L_{1} L_{2}^{2} \cos \left(u_{4}+\theta_{2}\right) & m_{22}=\frac{1}{3} m_{2} L_{2}^{3}
\end{array}
$$

Items of matrix $\mathrm{H}$ are:

$$
\begin{aligned}
& h_{11}=-\left(\dot{\theta}_{1} \dot{u}_{4}+\frac{1}{2} \dot{u}_{4}^{2}+\dot{\theta}_{2} \dot{u}_{4}+\dot{\theta}_{1} \dot{\theta}_{2}+\frac{1}{2} \dot{\theta}_{2}^{2}+\frac{1}{2} \dot{\theta}_{1}^{2}\right) m_{2} L_{2}^{2} \sin \left(u_{4}+\theta_{2}\right)-\frac{13}{35} m_{1} L_{1} u_{3} \dot{\theta}_{1}^{2}+\frac{11}{210} m_{1} L_{1}^{2} u_{4} \dot{\theta}_{1}^{2} \\
& -m_{2} L_{2} u_{3} \dot{\theta}_{1}^{2}-\frac{1}{L_{1}^{3}}\left(6 E I_{1} L_{1} u_{4}-12 E I_{1} u_{3}\right)+\left[\frac{7}{20} m_{1} L_{1}^{2}+\frac{1}{2} m_{2} L_{2}^{2} \cos \left(u_{4}+\theta_{2}\right)+m_{2} L_{1} L_{2}\right] \ddot{\theta}_{1}+\frac{1}{2} m_{2} L_{2}^{2} \cos \left(u_{4}+\theta_{2}\right) \ddot{\theta}_{2}
\end{aligned}
$$




$$
\begin{aligned}
& h_{21}=m_{2} L_{2}^{2} \dot{\theta}_{1} \dot{u}_{3} \sin \left(u_{4}+\theta_{2}\right)-\frac{1}{105} m_{1} L_{1}^{3} \dot{\theta}_{1}^{2} u_{4}+\frac{11}{210} m_{1} L_{1}^{2} \dot{\theta}_{1}^{2} u_{3}-\frac{1}{2} m_{2} L_{2}^{2} \dot{\theta}_{1}^{2} u_{3} \cos \left(u_{4}+\theta_{2}\right)+\frac{1}{2} m_{2} L_{1} L_{2}^{2} \dot{\theta}_{1}^{2} \sin \left(u_{4}+\theta_{2}\right) \\
& +4 E I_{1} u_{4} L_{1}-6 E I_{1} u_{3}-\left[\frac{1}{20} m_{1} L_{1}^{3}-\frac{1}{2} m_{2} L_{2}^{2} u_{3} \sin \left(u_{4}+\theta_{2}\right)+\frac{1}{3} m_{2} L_{2}^{3}-\frac{1}{2} m_{2} L_{1} L_{2}^{2} \cos \left(u_{4}+\theta_{2}\right)\right] \ddot{\theta}_{1}-\frac{1}{3} m_{2} L_{2}^{3} \ddot{\theta}_{2}
\end{aligned}
$$

From Fig.2 to Fig.3, results of model calculation are acquired through analyzing the trajectory of manipulator endpoint and deviation between $\mathrm{x}$ direction and $\mathrm{y}$ direction respectively, which is compared with reckoning of RecurDyn. It can be perceived that computations of the trajectory of manipulator endpoint of RecurDyn and this model are basically the same. The maximum deviation of $\mathrm{x}$ direction is $13.2 \mathrm{~mm}$ and $14.6 \mathrm{~mm}$ respectively. The maximum difference between them is $1.7 \mathrm{~mm}$ at the most inconsistent point. The maximum deviation of $\mathrm{y}$ direction is $18.2 \mathrm{~mm}$ and $22.3 \mathrm{~mm}$ respectively. The maximum difference between them is $4.5 \mathrm{~mm}$ at the most inconsistent point.

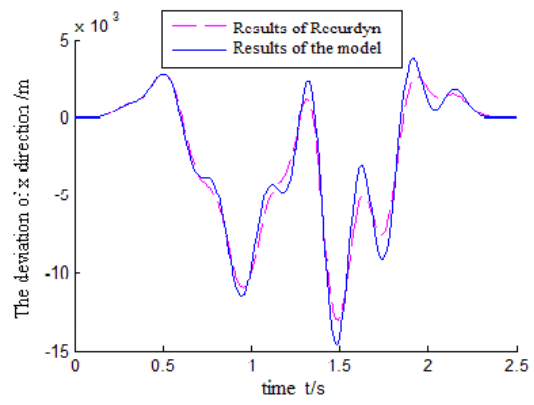

Fig.2. The deviation of $\mathrm{x}$ direction

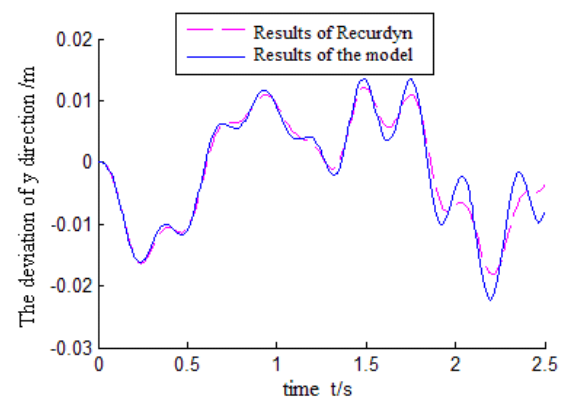

Fig.3. The deviation of y direction

\section{Conclusions}

Dynamics modeling and its Matlab symbol derivation are proposed for serial rigid-flexible manipulators in terms of Finite Element Method. In the process of dynamics modeling of manipulators, position and velocity of any node in the global coordinate system can be obtained on the basis of homogeneous coordinate and system dynamics model can be obtained on the basis of derivation of Lagrange approach. On this basis, symbolic expressions of system dynamics model can be acquired by means of Matlab symbol derivation method. The correctness and generality of the proposed modeling method has been fully validated through a number of simulation examples and the contrastive analysis of calculated results of multi-body dynamics analysis software RecurDyn. Efficient modeling and dynamic analysis of different types of rigid-flexible manipulators can be implemented by this modeling method.

\section{References}

[1] Tokhi M O, Azad K M. Flexible Robot manipulators: Modelling, Simulation and Control [M]. IET, 2008.

[2] Chen H Y, Xu D and Wang H. Three dimension curve welding seam modeling for seam tracking. 17th IFAC World Congress in Seoul Korea, 2008b.9686-9691.

[3] M Arteaga. On the properties of a dynamic model of flexible robot manipulators [J]. ASME Journal of Dynamic Systems, Measurement and Control, 1998120 (4) 8-14.

[4] A Mannani and H A Talebi. A fuzzy Lyapunov-based control strategy for a macro-micro manipulator: Experimental results. IEEE Trans. Control Syst. Technol, 2007.375-383.

[5] Z Jianbo, G Feng, Y Yi and L Wei. Dynamics Model on 6-PPPS 6-DOF Parallel Robot [J]. Machine Design and Research, 200723 (6) 27-30. 